\title{
Revisiting Binary Sequence Length Requirements to Accurately Emulate Optical Transmission Systems in Highly Dispersive Regime
}

\author{
Edouard Grellier*a ${ }^{\mathrm{a}}$,ean-Christophe Antona ${ }^{\mathrm{a}}$, Alberto Bononi ${ }^{\mathrm{b}}$, Sébastien Bigo ${ }^{\mathrm{a}}$ \\ ${ }^{a}$ Alcatel-Lucent, Bell-Labs, Route de Villejust, 91620 NOZAY - FRANCE; \\ ${ }^{\mathrm{b}}$ Dipartimento di Ingegneria dell' Informazione, Univ. di Parma, Viale G.P. Usberti, 43100, \\ Parma, Italy
}

\begin{abstract}
When increasing channel bit rate beyond $10 \mathrm{~Gb} / \mathrm{s}$ or when operating over fiber lines with sparse or no in-line dispersion compensation, Kerr-like non-linear effects can be considered as second order with respect to dispersive effects, because pulse broadening can expand over numerous neighbor pulses, before optical non-linear effects imprint their signature noticeably. To accurately emulate the interactions between pulses in this case, a few studies emphasized that PseudoRandom Binary Sequences (PRBS) should be used, with exponential dependence of the required PRBS length on bit rate and accumulated dispersion. In this paper, we explain our strategy to numerically estimate the required number of random, noisy bits for Monte-Carlo simulations, and show that it weakly increases in presence of pulse to pulse correlations and commonly tolerated levels of non-linearities (i.e. leading to transmission penalties as high as $1.5 \mathrm{~dB}$, for reference BERs of $10^{-2}, 10^{-3}$ or $10^{-5}$ ). Then we determine the actual required PRBS length that yields the same (sufficient) BER accuracy as the MC method. We demonstrate its actual dependence on BER, and show that MC theory provides a reliable upper bound in FEC-assisted, highly dispersive systems.
\end{abstract}

Keywords: Optical transmission, Chromatic dispersion, Monte-Carlo, PRBS, Sequence length

\section{INTRODUCTION}

When increasing channel bit rate beyond $10 \mathrm{~Gb} / \mathrm{s}$ or when operating over fiber lines with sparse or no in-line dispersion compensation, the transmission regime changes into the so-called highly dispersive (or pseudo-linear) regime [1],[2]. Thus, each pulse can expand over numerous neighbor pulses, and interact with them through optical non-linearities. For accurate emulation of these interactions, a few numerical studies recommend that Pseudo-Random Binary Sequences (PRBS) of sufficient length should be used [3]-[4], with exponential dependence on bit rate and accumulated dispersion. For instance, with transmission of non-return-to-zero (NRZ) channels at 40Gbit/s over 7x100km Standard Single Mode Fiber (SMF) without inline compensation, but with optimized dispersion compensation at both ends, the required PRBS length should be as large as $2^{39}$ or $2^{52}$ bits, according to models from [4] or [3] respectively. Managing such lengths would not only take unrealistically long simulation times, but also raise serious concerns about the accuracy of lab measurements with bit-error rate (BER) test sets. However, due to the widespread use of high-performance ForwardError Correction (FEC) techniques, the target BER generally lies between $10^{-2}$ and $10^{-6}$ before correction, which suggests revisiting Monte-Carlo-like simulations for emulating the performance of systems. In such simulations, it is generally accepted that the required number of random noisy bits is about 100/BER[5], i.e. $10^{4}-10^{8}$ for a relative error of $10 \%$, provided that neighbour pulses do not interact. Here, we focus on non-linear, highly dispersive systems where the samples have interacted and therefore become correlated across the transmission path. In this case the required number of bits is expected to be higher. Next, we numerically estimate the required number of random, noisy bits using the MC method versus the expected BER, in highly dispersive systems where pulse-to-pulse interactions are high. Then, we determine the actual PRBS length that yields the same (sufficient) BER accuracy as the MC method.

*edouard.grellier@alcatel-lucent.fr; phone+33 13077 7884; fax 1222 555-876; www.alcatel-lucent.com

Optical Transmission, Switching, and Subsystems VI, edited by Ken-ichi Kitayama, Pierpaolo C. Ghiggino, Kim Roberts, Yikai Su, Proc. of SPIE Vol. 7136, 713613 · C 2008 SPIE · CCC code: 0277-786X/08/\$18 · doi: 10.1117/12.803478 


\subsection{Existing models}

The conventional approach to study such systems is to emulate all the possible interactions between the neighboring pulses. This means that, if each pulse can interact with up to $M$ neighbors, all the possible pattern of length $M+1$ should be emulated. This is realized by using a Pseudo Random Binary Sequence of order $m=M+1$.

In [3], an estimation of the number of neighboring bits affected by the broadening of one pulse led to the requirement that the PRBS should be at least $2^{\mathrm{m}}-1$ bit-long, with ${ }_{m \geq C D_{\max }} \lambda^{2} B \frac{\Delta f}{c}+1$ to be representative of $\mathrm{m}$ bits interactions, where $\mathrm{CD}_{\max }$ is the maximum excursion of cumulated dispersion along a transmission link, B the bit-rate, $\Delta \mathrm{f}$ the spectral width of the considered modulation format, $\mathrm{c}$ the speed of light, and $\lambda$ the channel wavelength. The order of required PRBS is thus expected to vary proportionally to the product $\mathrm{CD}_{\max } \mathrm{B}^{2}$ for a given format.

In [4], an alternative estimate of the number of interacting neighbors of optimized periodic dispersion-managed transmission links based on a linearization of the Dispersion-Managed Non-Linear Schrödinger equation concluded that the order $\mathrm{m}$ of PRBS should be as large as $m \geq \frac{4 \pi d}{\sqrt{3}}\left(3 S^{2}+\xi_{i n}^{2}\right)^{1 / 4}$, with $\mathrm{d}$ the duty cycle, while $S=\frac{1}{2 \pi c}\left(\frac{\lambda B}{d}\right)^{2} \frac{L_{\text {span }} D_{\text {loc }}-D_{\text {res } / \text { span }}}{\alpha \cdot L_{\text {span }}}$ is the map strength $\left(\mathrm{D}_{\mathrm{loc}}\right.$ is the line fiber local dispersion, $\mathrm{D}_{\mathrm{res} / \mathrm{span}}$ the residual dispersion per span [6], $\mathrm{L}_{\text {span }}$ the span length, and $\alpha$ the fiber attenuation), and $\xi_{\text {in }}$ is the inline cumulated dispersion $\xi_{\text {in }}=\frac{1}{2 \pi c}\left(\frac{\lambda B}{d}\right)^{2} N_{\text {spans }} D_{\text {res/span }}$. Under such assumptions, the required PRBS order scales proportionally to the bit rate and square root of $\mathrm{CD}_{\max }$.

The relevance of both models was established numerically and it was shown that the required number of bits increases exponentially with the symbol rate and the excursion of cumulated dispersion. As an example, consider a $7 \times 100 \mathrm{~km}$ transmission at 40Gbit/s over Standard Single Mode Fiber (SSMF) without inline dispersion compensation for nonreturn-to-zero (NRZ) modulated channels, and optimized Pre- and Post-dispersion compensation at transmitter/receiver ends. In this case, the PRBS length should be as large as $2^{52}$ bits according to model from [3] (or $2^{39}$ if we use the formulae from [4] assuming it still holds without dispersion management).

\subsection{System under study}

In the following, the system under study consists of a transmission link composed of $7 \times 100 \mathrm{~km}$ standard single mode fiber $(17 \mathrm{ps} / \mathrm{nm} / \mathrm{km}$ local chromatic dispersion) with singly periodic dispersion maps similar to [6], for a single NRZ channel modulated at $43 \mathrm{~Gb} / \mathrm{s}$, with different values of Residual Dispersion Per Span (RDPS) between 0 (full inline compensation) and $1700 \mathrm{ps} / \mathrm{nm}$ (no inline compensation, most dispersive regime). The evolution of the chromatic dispersion during the propagation is represented on Fig. 1.For each configuration, we optimize pre- and post-dispersion compensation in the terminals, and vary fiber input power, so as to get the power corresponding to $1.5 \mathrm{~dB}$ Optical Signalto-Noise Ratio (OSNR) penalty at a reference BER (either $10^{-2}, 10^{-3}$ or $10^{-5}$ ). This power level, referred to as non-linear threshold (NLT) corresponds to a typical upper bound of operation in the non-linear regime. For the sake of simplicity, we overlook the impact of non-linearities in the dispersion compensating modules. At receiver end, noise is added voluntarily to reach the reference BER. The receiver is modeled by $0.5 \mathrm{~nm}$-bandwidth 2 nd order Gaussian optical filter, followed by a photodiode, a $28 \mathrm{GHz}$ bandwidth 5 th order Bessel electrical filter and an ideal decision gate.

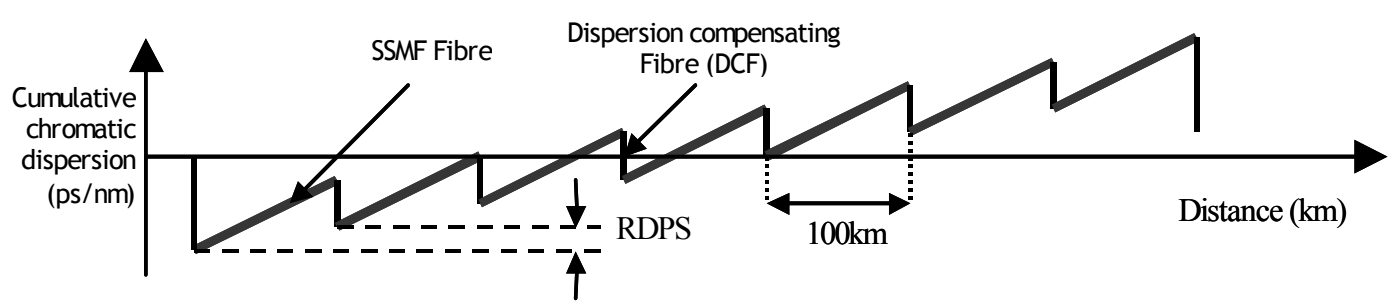

Fig. 1. Cumulative Chromatic dispersion along the transmission line 


\section{MONTE-CARLO METHOD : USING RANDOM SEQUENCES}

\subsection{Methodology}

Monte-Carlo simulations are achieved here by randomly drawing bit sequences and noise samples. The bit-error-rate is estimated by error counting, once a decision threshold and a decision time are set. Let us consider a single-channel binary transmission system. The BER is the probability $p$ of getting an error on any bit. We define the indicator of the error event $I_{j}=1$ if an error occurs on the $j$-th bit, or else $I_{j}=0$. Naturally, the expected value of $I_{j}$ is equal to $p$. In absence of correlations between the sampled signal and neighbouring bits, the number of noisy bits required to estimate a given BER with $10 \%$ relative error is about 100 /BER [5]. Here, we focus on systems where the samples have interacted and therefore become correlated across the transmission path. In this case, we explain our strategy for estimating the required number of noisy bits $\mathrm{N}$. In order to get $\mathrm{N}=\mathrm{LK}$ noisy bits, we perform $\mathrm{K}$ independent computation runs (thus $\mathrm{K}$ independent blocks) of $\mathrm{L}$ bits each, with $\mathrm{L}$ long enough with respect to the number of possibly interacting neighbours. For the investigated systems, values such as 256 bits are enough. For each block $k$ of L bits, we then derive an estimator of the BER $\bar{p}_{L}(k)=\frac{1}{L} \sum_{j=1}^{L} I_{j}(k)$, and deduce the total Monte Carlo BER estimate as $\hat{p}_{N}=(1 / K) \sum_{k=1}^{K} \bar{p}_{L}(k)$. An unbiased estimation of the variance of $\hat{p}_{N}$ is $\hat{\sigma}_{p}^{2}=\frac{1}{K(K-1)} \sum_{k=1}^{K}\left(\bar{p}_{L}(k)-\hat{p}_{N}\right)^{2}$. Finally, an estimation of the relative error is $\hat{\varepsilon}=\hat{\sigma}_{p} / \hat{p}_{N}$. The Monte-Carlo iterative process consists in repeating new simulations of $\mathrm{L}$ bits each until the estimation of the relative error becomes lower than $10 \%$.

\subsection{Validation}

To verify the reliability of the above procedure, we performed simulations without inline dispersion compensation, at the $\mathrm{NLT}$, i.e. $5.8 \mathrm{dBm}$, and $17.1 \mathrm{~dB}$ OSNR $/ 0.1 \mathrm{~nm}$ at the receiver. This ensures $\sim 10^{-3}$ BER after propagation. We ran the MC algorithm 100 times with different random seeds, and derived a standard deviation $\sigma$ of BER estimates, of $0.810^{-4}$, and thus 0.08 error, just below $10 \%$, as shown in Fig. 2. This confirms the validity of our stopping criterion on $\hat{\varepsilon}$.

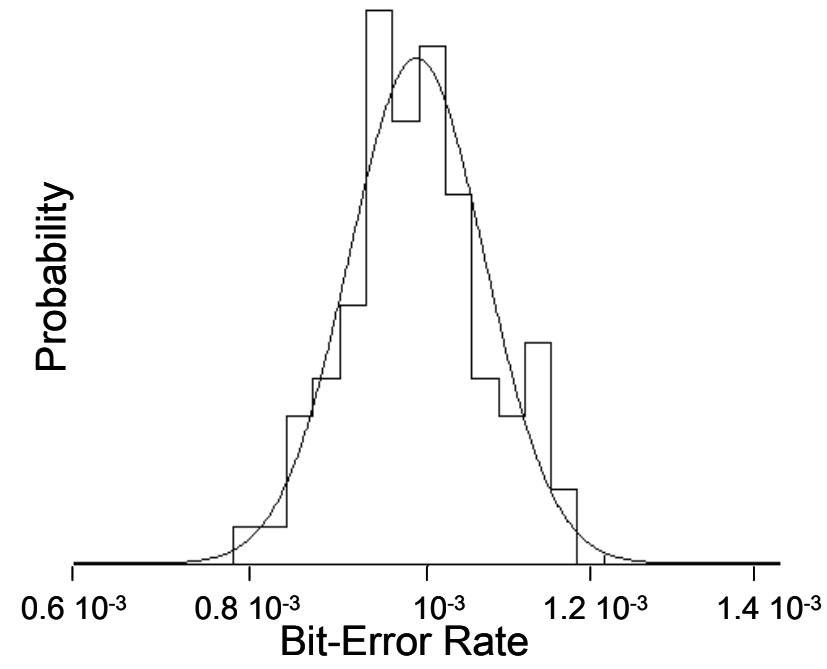

Fig. 2. eProbability density function of our Monte-Carlo BER estimation, for a 7x100km SSMF transmission link without inline dispersion compensation, at $5.8 \mathrm{dBm}$ fiber input power and $17.1 \mathrm{~dB}$ OSNR.

We then check whether our division process into blocks has a noticeable impact on the BER, as compared to what would be obtained from a single-block ultra-long sequence computed at once. Insight on this issue is given by varying the block size. For the same transmission link (i.e. no inline dispersion compensation, $5.8 \mathrm{dBm}$ inline fiber input power, and $17.1 \mathrm{~dB}$ 
OSNR $/ 0.1 \mathrm{~nm}$ ), we computed BERs assuming blocks of $\mathrm{K}=256, \mathrm{~K}=512, \mathrm{~K}=1024$, and $\mathrm{K}=2048$ bits. Not only we obtained the same BER whatever the size block (BER=1.04e-3, 1.02e-3, 1.05e-3, 1.02e-3 for K=256, 512, 1024, 2048), but also the same number of bits was required to meet the stopping criterion $(\mathrm{N}=102400,100352,101376,98304$ for $\mathrm{K}=256,512,1024,2048)$.

Finally, we compared the results obtained by our Monte-Carlo procedure and results obtained using a Pseudo Random Binary Sequence of 32768 bits, for a transmission link with full inline dispersion compensation, in which case the PRBS length chosen is enough according to models [3] and [4]. Both methods (Monte-Carlo, and using a PRBS sequence of 32768 bits) were in good agreement, in particular they lead to the same value of the non linear threshold i.e. $7 \mathrm{dBm}$.

\section{PERFORMANCES OF THE MONTE-CARLO METHOD}

We are here interested in the number $N$ of noisy bits required to obtain a 0.1 relative error. In absence of correlation, the theory predicts that this number is equal to 100/BER [5]. In this section, we try to determine how this number varies in an actual system with possible correlations between neighboring bits.

\subsection{Influence of the OSNR}

For different transmission links, we vary the OSNR, therefore changing the bit error rate, and observe the number of bits corresponding to $\hat{\varepsilon}=0.1$. It was found that considering $N$ as a function of $1 / B E R$ was more interesting than considering it as a function of the OSNR. In fact the variation of the BER remains the main impact of the OSNR on the required number of bits to obtain a 0.1 relative error.

On Fig. 3 is reported the number of bits corresponding to $\hat{\varepsilon}=0.1$ for several transmission links : a back-to-back transmission, a transmission with full inline compensation and a transmission without inline compensation

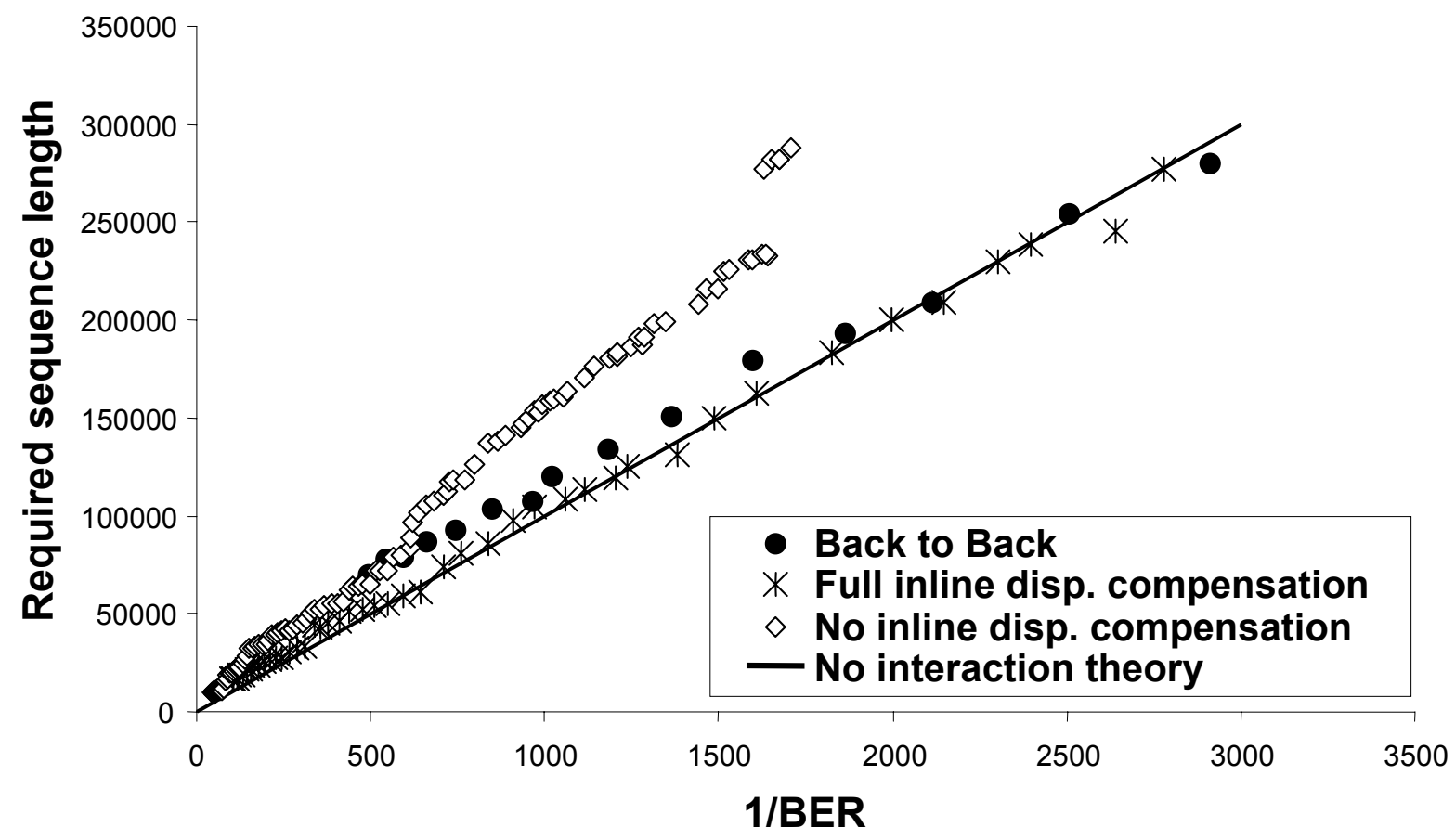

Fig. 3. Number of bits required for a 0.1 estimated relative error. Comparison between the theory without correlations between neighboring bits, and numerical results for the following transmission : back-to-back, no inline dispersion compensation, full inline dispersion compensation, the power input of inline fibers is set to $7 \mathrm{dBm}$ (The NLT for reference BER $=10^{-3}$ is $7 \mathrm{dBm}$ and $5.8 \mathrm{dBm}$ for the case of full inline compensation and no inline compensation respectively). 
We observe that both the back-to-back and the full inline dispersion compensation cases are in good agreement with the theory without correlation. However, with no inline dispersion compensation, the required number of bits is larger than predicted by the above theory. These results show that Monte-Carlo convergence is affected by the correlations due to the bit-to-bit interactions in the highly dispersive regime.

We can note that whatever the transmission link, the required number of bits tends to scale as the inverse of the BER. Thus we can define N/NO the ratio between the actual number of bits required, and the required number of bits in the case with no correlation.

\subsection{Influence of the dispersion map}

As the cumulated dispersion increases, each pulse expands over more and more neighboring pulses, and therefore the number of possible correlations increases. On Fig. 4 is represented the evolution of the N/NO ratio as the residual dispersion per span increases, and the inline fiber input power is fixed at $7 \mathrm{dBm}$ (i.e. the non linear threshold at $10^{-3}$ biterror-rate with full inline dispersion compensation).

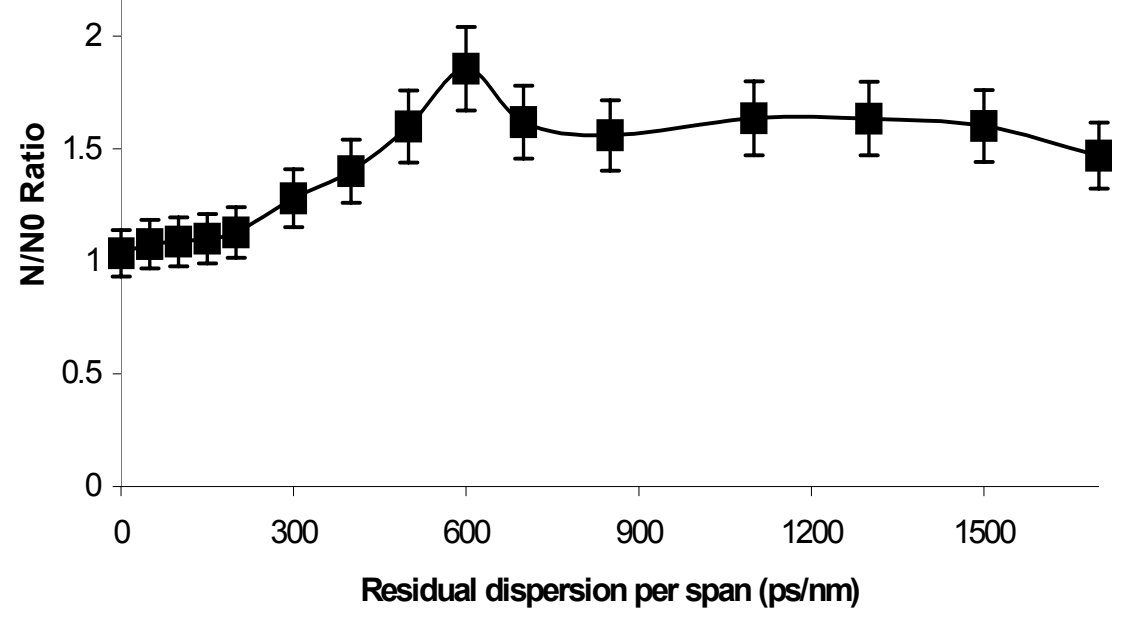

Fig. 4. N/N0 ratio over the residual dispersion per span. The fiber input power is $7 \mathrm{dBm}$

For the chosen inline fiber power, the number of bit required is 100/BER for full inline transmission (as predicted by the "no correlation theory"). As the residual dispersion per span increases (up to 600ps/nm), the amount of inter-symbol correlation becomes larger, and ratio $N / N O$ increases.

However, this behavior doesn't continue for larger residual dispersion per span (600ps/ $\mathrm{nm}$ in our case) even if the number of possible interactions increased. The increase in the number of interactions is probably balanced by a decrease of the strength of those interactions.

\subsection{Influence of the power}

The importance of non linear effects increases with the inline fiber power. Even if the power doesn't directly change the number of neighboring pulses interacting, it modifies the importance of the interactions. That's why the required number of bits is expected to increase with the power. Fig. 5 represents the evolution of the N/NO ratio in the case of a transmission without inline dispersion compensation. The $N / N O$ ratio is roughly equal to 1 for power values up to $6 \mathrm{dBm}$ and then increases significantly. This increase corresponds roughly to the non linear threshold at $10^{-3} \mathrm{BER}$, (i.e. $5.8 \mathrm{dBm}$ in this case). Similar results have been observed for all dispersion maps studied : the correlations between the errors only slow down the convergence of Monte-Carlo for power larger than the non linear threshold at $10^{-3} \mathrm{BER}$. 


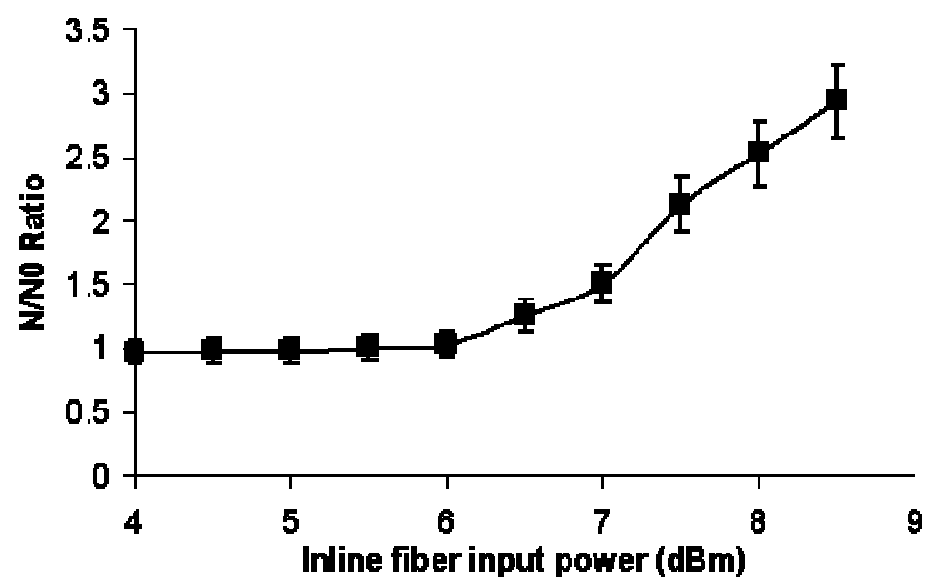

Fig. 5. N/N0 ratio over the inline fiber input power for a transmission of 7x100km of SSMF with no inline compensation.

\subsection{Summary}

The interactions between pulses change the number of bits required for the Monte-Carlo convergence. However, in the "worst case" studied (no inline dispersion compensation and fiber input power $2.5 \mathrm{~dB}$ over the NLT at $10^{-3}$ BER) the required number of bits is only 3 times the one predicted by the theory without correlation (ie. $N / N O=3$ ), this factor 3 is negligible in comparison with the exponential dependence of [3] and [4]. Plus, the cases where the N/NO ratio is significantly larger than 1 correspond to power values well above the non linear threshold, that have little interest and leads to high BER, thus the Monte-Carlo convergence is fast in those cases despite the high N/NO ratio.

We can therefore conclude that for the transmission links under study, the correlations between bits doesn't slow down significantly the Monte-Carlo computation.

Finally if we consider a FEC-assisted system operating at $10^{-3} \mathrm{BER}$, the required length of the Monte-Carlo method is $\sim 10^{5}$ even in the most dispersive regime studied, i.e. several order of magnitude under the required sequence length predicted by previous models ( $10^{11}$ and $10^{15}$ bits required according to models [4] and [3] respectively)

\section{REQUIRED PRBS LENGTH TO EMULATE FEC-ASSISTED SYSTEMS}

We have seen with Monte-Carlo that it wasn't necessary to emulate all the possible interactions, and that the bit-errorrate was a key element for the required length of a random sequence.

On the other hand, in simulations as well as in test equipments in the laboratories, PRBS are usually used rather than random sequences. Previous models studying the required PRBS length considered that all the possible interactions should be emulated. In this section we study the actual required length of a pseudo random binary sequence to assess an accurate BER estimation, in systems operating at the high BERs enabled by the FEC.

Next, we compare the requirements of the above MC method with the so-called PRBS method. Contrary to MC, the PRBS method resorts to pre-defined bit-sequence and uses semi-analytical BER estimation (provided by KarhunenLoeve [7]). The actual type of bit sequence used is De Bruijn bit sequence, i.e. a PRBS with an additional " 0 " bit inserted in the longest run of zeros.

\subsection{Method to evaluate the required PRBS length}

Contrary to Monte-Carlo, while using a pseudo random binary sequence, we don't have an estimator of the relative error. Our strategy to evaluate the required PRBS length is to performed simulations with PRBS of increasing length until we get the same values of the non linear thresholds for different residual dispersion per span, with several consecutive PRBS orders. 
To verify the reliability of this procedure we applied it to a random sequence. We have seen in section 3 , that the requirement of our Monte-Carlo procedure to assess the non linear threshold at $10^{-3}$ BER was $\sim 10^{5}$ bits. The results are reported on Fig. 6, when the sequence length is half of the required length (i.e. 50000 bits), we can observe errors of 0.2 $\mathrm{dB}$ for some values of residual dispersion per span (here 1300 and $1500 \mathrm{ps} / \mathrm{nm}$ ). This procedure therefore enables to witness the inaccuracy of a sequence that is only half of the required length, this way we can assess the PRBS length that yields the same accuracy as the Monte-Carlo method.

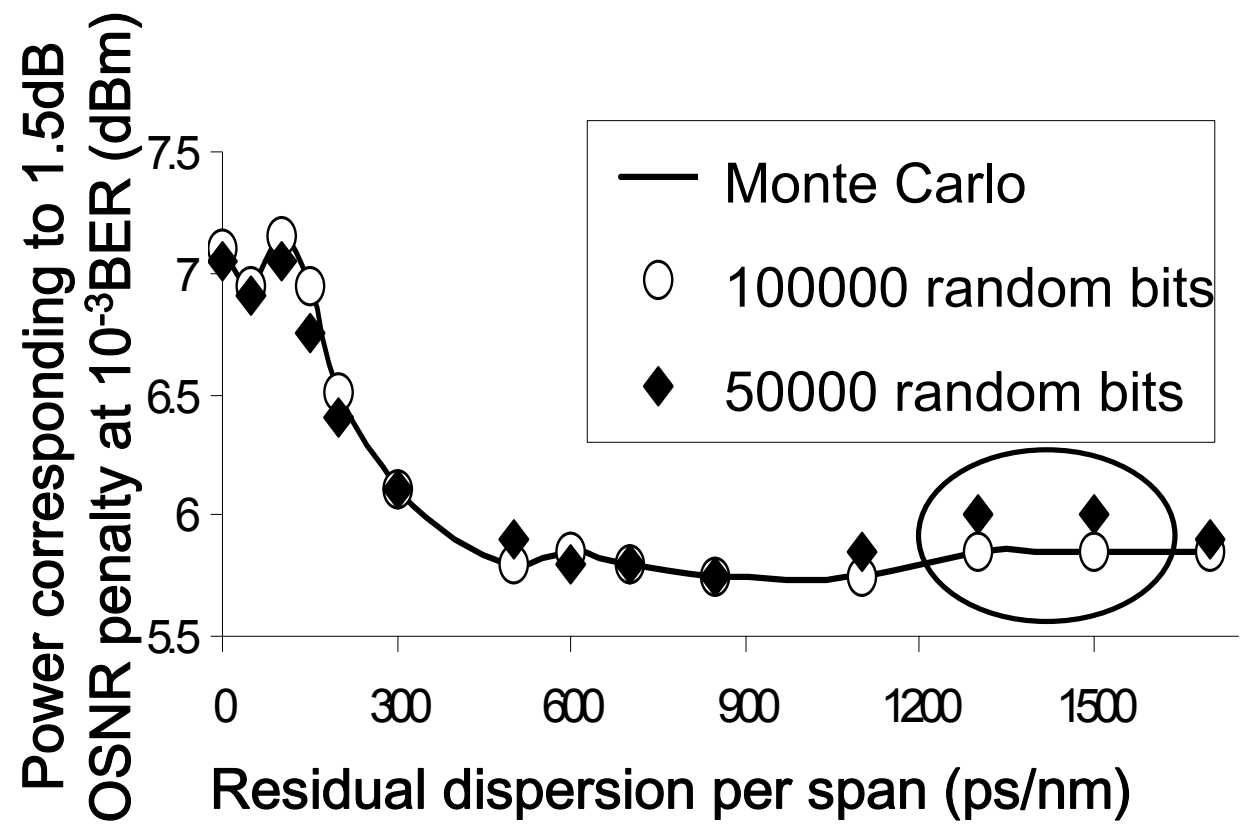

Fig. 6. Non linear thresholds at $10^{-3}$ BER obtained using random sequences of 50000 bits, 100000 bits and using our Monte-Carlo stop criterion, for different values of residual dispersion per span.

\subsection{Required PRBS length}

Fig. 7 represents the non linear threshold versus the residual dispersion per span for MC simulations, and PRBS simulations with variable sequence lengths, for a reference BER of $10^{-3}$. While accurate MC simulations require $\sim 100$ /BER whatever the residual dispersion per span. The PRBS lengths required to provide the same accuracy as MC are much smaller but should increase with the residual dispersion per span (for example a PRBS of 2048 bits is enough for residual dispersion per span smaller than $300 \mathrm{ps} / \mathrm{nm}$ but not for larger values). Otherwise, the NLT departs from MC predictions. At this $10^{-3}$ BER, the PRBS length needs not exceed 8192 bits, despite strong inter-symbol interactions. This value is well smaller than the predictions of theories of [3] and [4].

The required PRBS length is found to depend on the BER, even if a 8192 bits long PRBS is enough to assess the non linear threshold at $10^{-3}$ BER , a PRBS of 16384 bits is still not enough at $10^{-5}$ BER of as illustrated on Fig. 8. In fact, at low BER, the receiver performance is much more affected by rare patterns and the required length is larger for either a random sequence or a PRBS. 


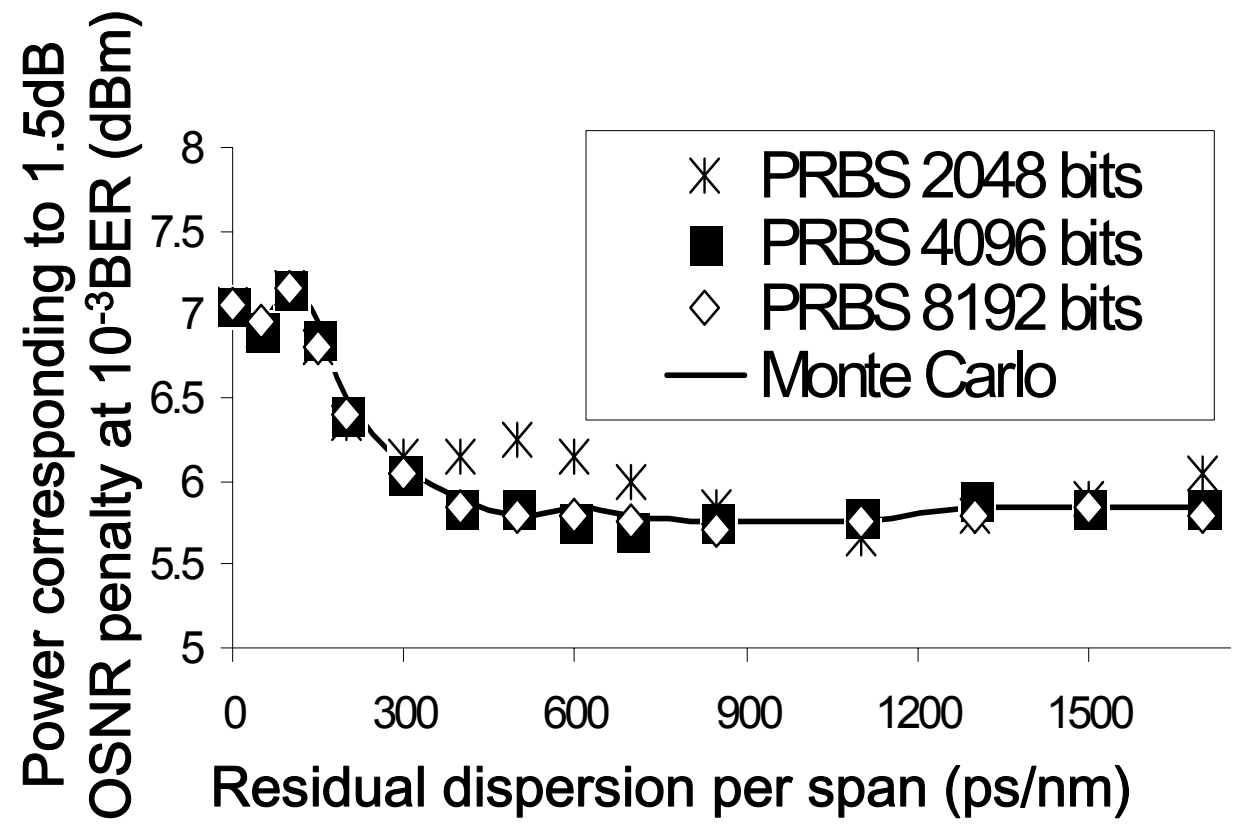

Fig. 7. Non linear thresholds at $10^{-3}$ BER obtained using PRBS of 2048, 4096, 8192 bits, and using our Monte-Carlo procedure, for different values of residual dispersion per span.

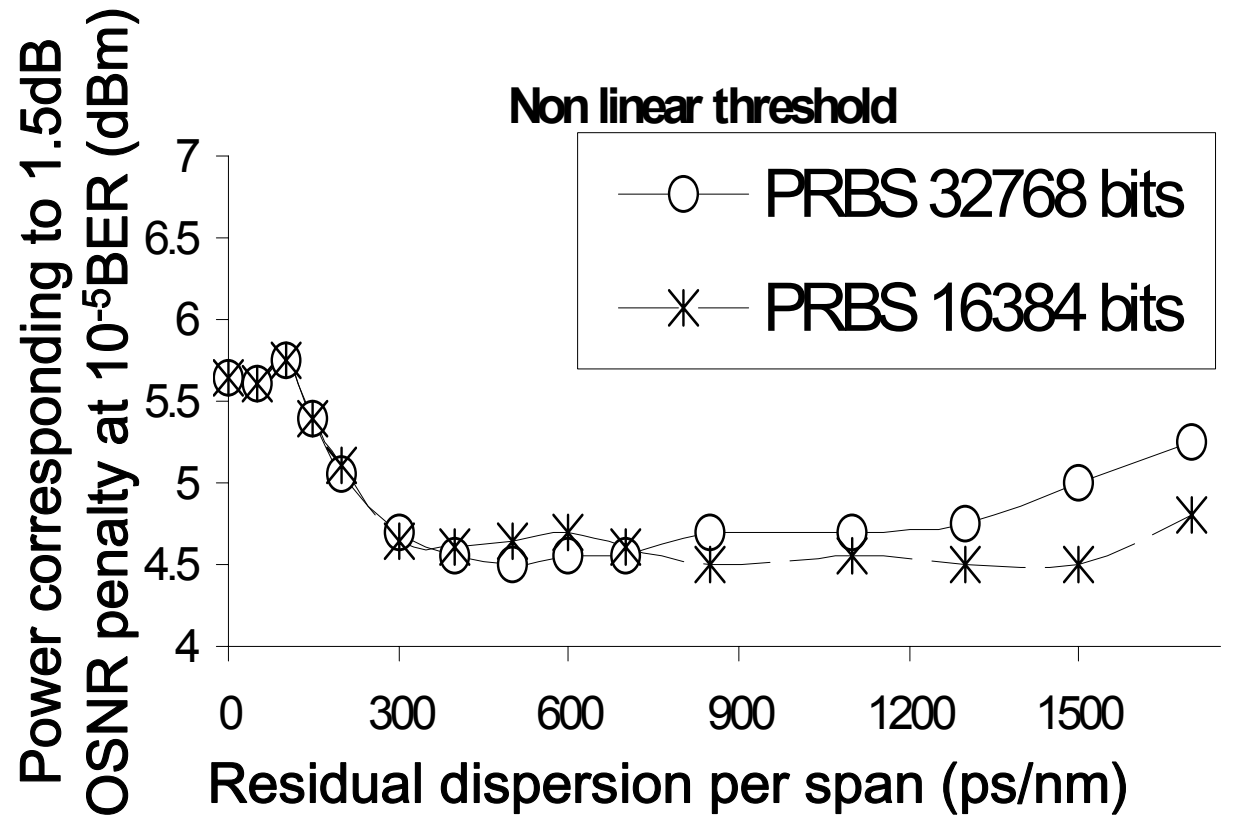

Fig. 8. Non linear thresholds at $10^{-5}$ BER obtained using PRBS of 16384 and 32768 bits, for different values of residual dispersion per span. 


\subsection{Comparison with Monte-Carlo method}

The work of Fig. 7 has been replicated at reference BERs $10^{-2}, 10^{-3}$ and $10^{-5}$ and the required sequence lengths derived. Fig. 9 summarizes the results. It depicts the evolution of the minimum required number of bits for MC and PRBS simulations versus the residual dispersion per span. This number is found almost constant for MC simulations and close to the expectation from [5], regardless of the residual dispersion per span, and thus inter-symbol correlations. In contrast, when the residual dispersion per span is relatively small, it can be seen that PRBS length requirements increase with the residual dispersion per span, in accordance with [4], even though slightly smaller. At larger values of residual dispersion per span, when requirements from models [3] and [4] become comparable or higher to MC requirements for an expected BER, the PRBS length requirements prove to depend also on the expected BER, but stabilize to an asymptotic value which is smaller than MC requirements. For instance, with expected (but realistic, considering the use of FEC) BER of $10^{-3}$, no more than $2^{13}$ bits (8192) are actually required, no matter the dispersion regime, far from the $2^{39}-2^{52}$ bitspredictions of [3]-[4]. Indeed, the latter overlook the finite value of BER and that it does not need to be estimated with less than $10 \%$ accuracy. It should be emphasized that this conclusion applies to typical pre-FEC BERs. From the above results, it can be extrapolated that BER estimates or measurements with at least $10 \%$ accuracy at $10^{-9}$ would require more than $2^{31}$ bit lengths in the highly dispersive regime.

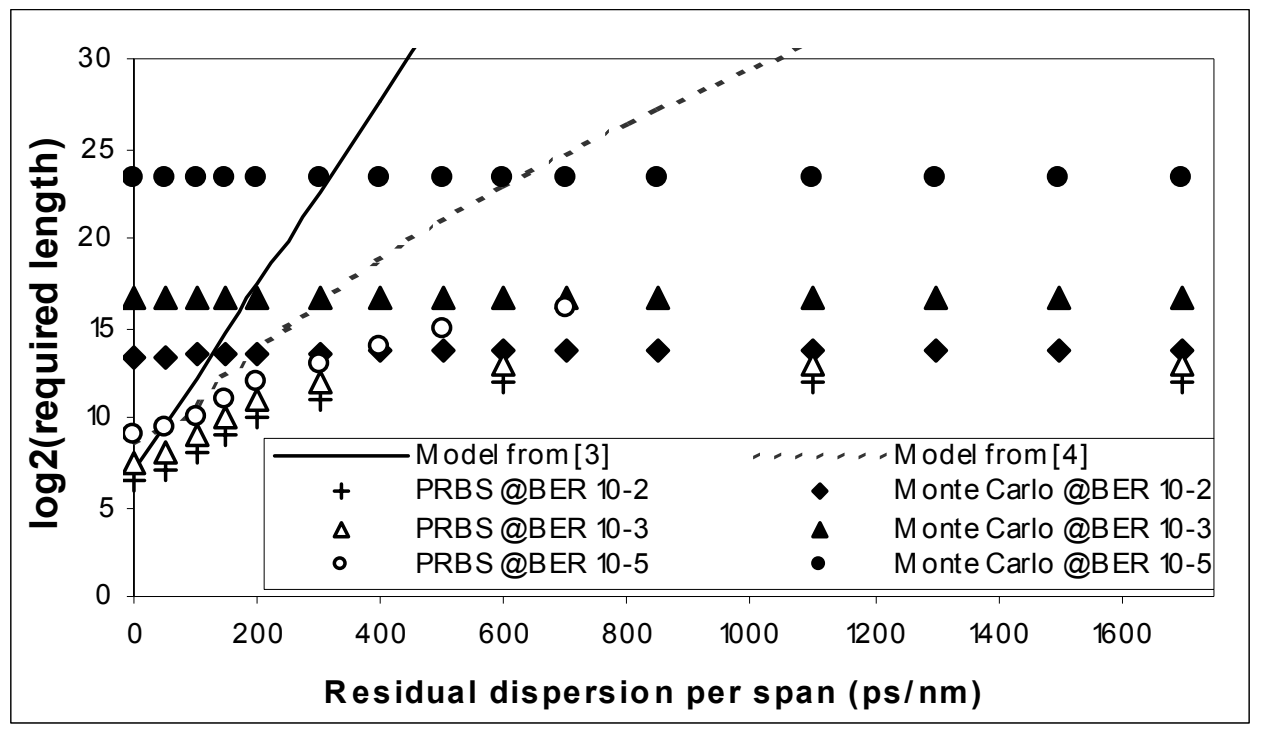

Fig. 9. Required number of bits. Comparison between models emulating all pulses interactions, and numerical result of requirements at non-linear threshold for various reference BERs, using MC or PRBS methods.

\section{CONCLUSION}

We demonstrated that the number of bits needed to run Monte-Carlo simulations weakly depends on correlations between neighboring bits in non-linear, highly dispersive transmission systems. Monte-Carlo theory was found to provide a reliable upper bound for sequence lengths in FEC-assisted, highly dispersive systems.

\section{REFERENCES}

[1] D. Van Den Borne et al, ECOC, Mo3.2.2, 2006

[2] C. Laperle et al, OFC'07, PDP 16, Anaheim (2007)

[3] L. K. Wickham et al, PTL., vol 16 (2004), p. 1591

[4] P. Serena et al, Proc. ECOC, We.3.P093, 2007. 
[5] G. Fishman, "Monte Carlo Concepts, Algorithms and Applications, NY, USA, Springer, 4th ed., 2003.

[6] J.-C. Antona et al, OFC'02, Anaheim, WX5, (2002)

[7] E. Forestieri, JLT, vol. 18, nº.11, p 1493, Nov. 2000 